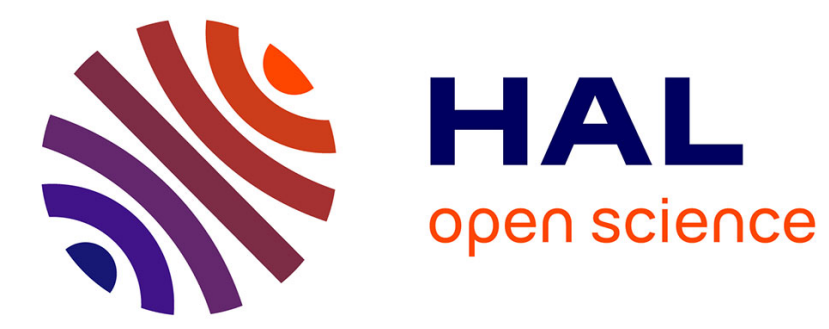

\title{
Traffic Matrix Reloaded: Impact of Routing Changes
}

\author{
Renata Teixeira, Nick Duffield, Jennifer Rexford, Matthew Roughan
}

\section{To cite this version:}

Renata Teixeira, Nick Duffield, Jennifer Rexford, Matthew Roughan. Traffic Matrix Reloaded: Impact of Routing Changes. Passive and Active Network Measurement, Mar 2005, Boston, United States. pp.251 - 264, 10.1007/978-3-540-31966-5_20. hal-01097542

\section{HAL Id: hal-01097542 \\ https://hal.inria.fr/hal-01097542}

Submitted on 19 Dec 2014

HAL is a multi-disciplinary open access archive for the deposit and dissemination of scientific research documents, whether they are published or not. The documents may come from teaching and research institutions in France or abroad, or from public or private research centers.
L'archive ouverte pluridisciplinaire HAL, est destinée au dépôt et à la diffusion de documents scientifiques de niveau recherche, publiés ou non, émanant des établissements d'enseignement et de recherche français ou étrangers, des laboratoires publics ou privés. 


\title{
Traffic Matrix Reloaded: Impact of Routing Changes
}

\author{
Renata Teixeira ${ }^{1}$, Nick Duffield $^{2}$, Jennifer Rexford $^{2}$, and Matthew Roughan ${ }^{3}$ \\ 1 U. California-San Diego, \\ teixeiradcs.ucsd.edu \\ 2 AT\&T Labs-Research, \\ \{duffield, jrex\}@research.att.com \\ 3 University of Adelaide \\ matthew.roughan@adelaide.edu.au
}

\begin{abstract}
A traffic matrix represents the load from each ingress point to each egress point in an IP network. Although networks are engineered to tolerate some variation in the traffic matrix, large changes can lead to congested links and poor performance. The variations in the traffic matrix are caused by statistical fluctuations in the traffic entering the network and shifts in where the traffic leaves the network. For an accurate view of how the traffic matrix evolves over time, we combine fine-grained traffic measurements with a continuous view of routing, including changes in the egress points. Our approach is in sharp contrast to previous work that either inferred the traffic matrix from link-load statistics or computed it using periodic snapshots of routing tables. Analyzing seven months of data from eight vantage points in a large Internet Service Provider (ISP) network, we show that routing changes are responsible for the majority of the large traffic variations. In addition, we identify the shifts caused by internal routing changes and show that these events are responsible for the largest traffic shifts. We discuss the implications of our findings on the accuracy of previous work on traffic matrix estimation and analysis.
\end{abstract}

\section{Introduction}

The design and operation of IP networks depends on a good understanding of the offered traffic. Internet Service Providers (ISPs) usually represent the traffic as a matrix of load from each ingress point to each egress point over a particular time interval. Although well-provisioned networks are designed to tolerate some fluctuation in the traffic matrix, large variations break the assumptions used in most designs. In this paper, we investigate the causes of the traffic matrix variations. Identifying the reasons for these disruptions is an essential step toward predicting and planning for their occurrence, reacting to them more effectively, or avoiding them entirely.

The traffic matrix is the composition of the traffic demands and the egress point selection. We represent the traffic demands during a time interval $t$ as a matrix $V(\cdot, \cdot, t)$, where each element $V(i, p, t)$ represents the volume of traffic entering at ingress router $i$ and headed toward a destination prefix $p$. Each ingress router selects the egress point for each destination prefix using the Border Gateway Protocol (BGP). We represent the 
BGP routing choice as a mapping $\varepsilon$ from a prefix to an egress point, where $\varepsilon(i, p, t)$ represents the egress router chosen by ingress router $i$ for sending traffic toward destination $p$. At time $t$ each element of the traffic matrix $\mathcal{T} M$ is defined as:

$$
\mathcal{T} M(i, e, t)=\sum_{p \in P: \varepsilon(i, p, t)=e} V(i, p, t) .
$$

where $P$ is the set of all destination prefixes.

Figure 1 presents a simple network with one ingress router $i$, two egress routers $e$ and $e^{\prime}$, and two external destination prefixes $p_{1}$ and $p_{2}$. Given traffic demands $V\left(i, p_{1}, t\right)$ and $V\left(i, p_{2}, t\right)$ and a prefix-to-egress mapping $\varepsilon\left(i, p_{1}, t\right)=\varepsilon\left(i, p_{2}, t\right)=e$, the traffic matrix for this network is $\mathcal{T} M(i, e, t)=V\left(i, p_{1}, t\right)+V\left(i, p_{2}, t\right)$ and $\mathcal{T} M\left(i, e^{\prime}, t\right)=0$.

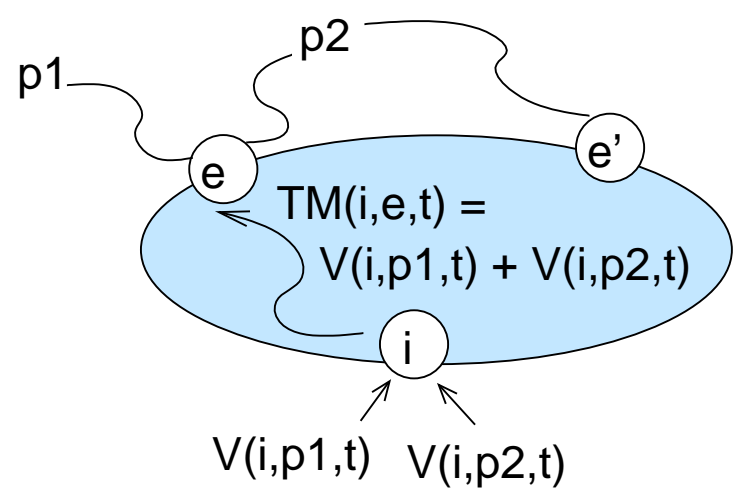

Fig. 1. Example of traffic matrix.

Fluctuations in the traffic demands and changes in the prefix-to-egress mapping cause the traffic matrix to vary. This paper considers the natural question: what are the causes of large variations in the traffic matrix?

Most previous work on measuring [1-4] and analyzing traffic matrices [5,6] has assumed that the prefix-to-egress mapping $\varepsilon$ is stable. However, relying on periodic snapshots of routing data runs the risk of associating some traffic measurements with the wrong elements in the traffic matrix, obscuring real variations in the traffic. In this paper, we study how changes in $\varepsilon$ impact the traffic matrix. A previous analysis of five traces of 6-22 hours in duration on the Sprint network [7] shows that most BGP routing changes do not lead to large traffic shifts. However, given that large traffic variations are infrequent (yet significant) events, we believe that longer traces are necessary to draw meaningful conclusions. Our previous work [10] shows that internal routing can cause $\varepsilon$ to change for a large number of prefixes at the same time, which can potentially cause a large traffic shift. Neither [7] nor [10] study the significance of traffic shifts caused by routing relative to regular traffic fluctuations, which is the topic of this paper. 
In this paper, we study the impact of routing changes on the traffic matrix over a seven-month period in a tier-1 ISP network. Using Cisco's Sampled Netflow feature [8] and feeds of internal BGP (iBGP) messages, we compute the traffic demands $V$ and the prefix-to-egress mapping $\varepsilon$ for eight ingress routers. Joining these two datasets allows us to construct a detailed view of the variation of the traffic matrix over time. We also collect measurements of the intradomain routing protocol [9] in order to identify the changes in $\varepsilon$ that were caused by internal network events, using the algorithm described in [10]. Our analysis shows that:

1. Although the likelihood of large traffic fluctuations is small, big changes do sometimes occur. In any given ten-minute time interval, less than $0.02 \%$ of the traffic matrix elements studied have a traffic variation of more than 4 times the normal traffic variations. However, some elements vary by more than 4 times the normal variations several times a week.

2. Most routing changes do not cause much variation in the traffic matrix. Previous studies $[7,11]$ have shown that routing changes typically do not cause large traffic shifts; most BGP routing changes affect destination prefixes that receive very little traffic.

3. Routing changes are responsible for many of the large traffic shifts: $58.6 \%$ of instances where a traffic matrix element fluctuated by more than 10 times the normal variation for that element could be explained by a BGP routing change.

Although routing changes usually do not affect much of the traffic, many of the large traffic shifts are triggered by routing changes. Large traffic shifts caused by routing are rare, but important events. After introducing our measurement methodology in Section 2, we identify the causes of the big variations in Section 3. Section 4 discusses the implications of our results on other studies of traffic matrices. Section 5 concludes the paper.

\section{Measuring Traffi c Matrix Variation}

Studying the variation of traffic matrix elements over time requires collecting finegrained measurements of traffic and routing. We analyze data collected from a tier-1 ISP network for 173 days from March to September 2004. We collect data from eight aggregation routers that receive traffic from customers destined to peers and other customers. The eight routers are located in major Points of Presence (PoPs) that are spread throughout the United States.

We compute eight rows of the traffic matrix, considering all traffic from these eight ingress aggregation routers to all of the egress PoPs. This section describes how we compute the prefix-to-egress mapping $\varepsilon(i, p, t)$ from the BGP data and the traffic demands $V(i, p, t)$ from the Netflow data. Once we have computed $\varepsilon$ and $V$, we use Equation 1 to compute the elements of the traffic matrix $\mathcal{T} M(i, e, t)$. The BGP monitor and the Netflow collection servers are NTP-synchronized, allowing us to use the timestamps to join the two datasets. 


\subsection{Prefix-to-Egress Mapping}

A BGP monitor collects internal BGP update messages directly from each vantage point. Configured as a route-reflector client of each vantage point, the BGP monitor receives updates reporting any change in the best BGP route at each router for each destination prefix. The monitor records each BGP update with a timestamp at the onesecond granularity.

A single network event, such as a failure or policy change, can lead to a burst of BGP updates messages as the routers explore alternate paths. Rather than studying the details of routing convergence, our analysis focuses on the changes from one stable route to another. Similar to previous studies $[10,11]$, we group the BGP updates for the same destination prefix that have an interarrival time of 70 seconds or less. Our analysis considers the stable route that existed before the flurry of updates and the new stable route that exists at the end.

Based on an initial BGP table dump and a sequence of BGP updates, we generate the prefix-to-egress mapping $\varepsilon(i, p, t)$ for any given time. The egress point corresponds to a $P o P$ rather than a specific router. We associate each egress router with a PoP based on the router name and configuration data.

\subsection{Traffic Demands}

Every vantage point has the Cisco's Sampled Netflow feature [8] enabled on all links that connect to access routers and exports flow records to a collection server at the same location. The collection server samples the flow records using the technique presented in [12] in order to reduce processing overhead, and computes 10-minute aggregated traffic volumes for each destination prefix. We use these aggregated reports to extract $V(i, p, t)$ for each vantage point $i$ and destination prefix $p$ at every 10-minute interval. Consequently, a reference to a time $t$ indicates the end of a 10-minute interval.

Because of sampling, the volumes $V(i, p, t)$ are random quantities that depend on the sampling outcomes. Through a renormalization applied to the bytes reported in sampled flow records, the quantities $V(i, p, t)$ are actually unbiased estimators of the volumes of the original traffic from which they were sampled, i.e., their average over all possible sampling outcomes is the original volume. The standard error associated with an aggregate of size $V$ is bounded above by $\sqrt{k / V}$ for some constant $k$ that depends on the sampling parameters [12]. For the parameters employed in the current case, $k<21 \mathrm{MB}$. Note that the standard error bound decreases as the size of the aggregate increases. This property aligns well with our focus on the largest changes in traffic rates: these are the most reliably estimated. As an example, for a 10-minute aggregate of traffic at a rate of $10 \mathrm{MB}$ per second, the standard error due to sampling is no more than $6 \%$.

Even though the traffic data is divided into 10-minute intervals, our 70 -second grouping of BGP updates is important for cases when path exploration crosses the boundary between two ten-minute intervals. This ensures that we focus our analysis on stable changes of $\varepsilon$. If the mapping $\varepsilon(i, p, t)$ changes more than once in a 10-minute interval, then we cannot distinguish the volume of traffic affected by each of them individually. Therefore, we exclude those cases from our analysis by ignoring intervals 
with prefixes that have more than one stable routing changes in that bin; this excludes $0.05 \%$ of the $(i, e, t)$ tuples from our study. We also exclude all traffic for the small number of flows that had no matching destination prefix in the BGP routing tables or update messages; we verified that these flows corresponded to an infinitesimal fraction of the traffic.

\section{Causes of Large Traffic Variations}

In this section, we explore the contributions of changes in the traffic demands $V$ and prefix-to-egress mapping $\varepsilon$ to the variations in the traffic matrix elements $\mathcal{T} \mathcal{M}$. Our analysis shows that, although most changes in $\varepsilon$ have a small effect on the traffic matrix, many of the large variations in the traffic matrix are caused by changes in $\varepsilon$. Also, we show that, while most changes in $\varepsilon$ are caused by external routing events, the small number of internal routing events are more likely to cause larger shifts in traffic.

\subsection{Definition of Traffic Variations}

Figure 2 shows an example of how two traffic matrix elements (with the same ingress point $i$ ) change over the course of a day. The total traffic entering at the ingress point varies throughout the day, following a typical diurnal cycle. For the most part, the traffic $\mathcal{T} M\left(i, e_{1}, t\right)$ has the same pattern, keeping the proportion of traffic destined to $e_{1}$ relatively constant. For most of the day, no traffic travels from ingress $i$ to egress point $e_{2}$. The most significant change in the two traffic matrix elements occurs near the end of the graph. The traffic leaving via egress point $e_{1}$ suddenly decreases and, at the same time, traffic leaving via egress point $e_{2}$ increases. This shift occurred because a routing change caused most of the traffic with egress point $e_{1}$ to shift to egress point $e_{2}$. The egress point $e_{2}$ also starts receiving traffic that had previously used other egress points (not shown in the graph), resulting in an increase for $e_{2}$ that exceeds the decrease for $e_{1}$. In the meantime, the total traffic entering the network at ingress $i$ remained nearly constant.

The traffic experiences other relatively large downward spikes (labeled as load variation). These spikes may very well be associated with a routing change in another AS in the Internet that caused traffic to enter at a different PoP (this kind of traffic variation was called an "ingress-shift anomaly" in [6]). In this paper, we analyze traffic shifts caused by routing changes experienced by our network. Finding a signature of routinginduced traffic variations for one network is an important first step to infer other traffic variations that are caused by routing changes in other networks.

To analyze these kinds of traffic fluctuations, we define the variation of a traffic matrix element at an interval $t$ as:

$$
\Delta \mathcal{T} M(i, e, t)=\mathcal{T} M(i, e, t)-\mathcal{T} M(i, e, t-1) .
$$

\subsection{Changes in Traffic Demands vs. Egress Points}

The variation of a traffic matrix element $(\Delta \mathcal{T} M)$ is composed of the load variation $(\Delta L)$, which represents volume fluctuations on the traffic demands $V$, and the routing 


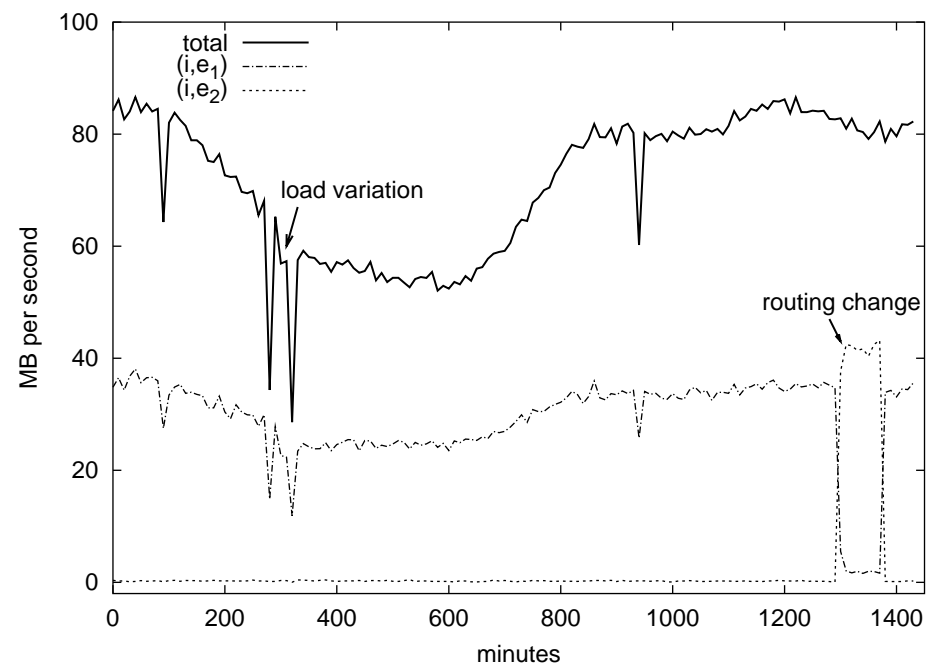

Fig. 2. Sample traffic volume from one ingress to two egresses.

shifts $(\Delta R)$, which accounts for changes in the prefix-to-egress mapping $\varepsilon$ :

$$
\Delta \mathcal{T} M(i, e, t)=\Delta L(i, e, t)+\Delta R(i, e, t)
$$

$\Delta L(i, e, t)$ represents the change in the volume of traffic for all destination prefixes that did not change their egress point from the previous time interval (i.e., $\varepsilon(i, p, t)=$ $\varepsilon(i, p, t-1)=e)$ :

$$
\Delta L(i, e, t)=\sum_{\substack{p \in P \\ \varepsilon(i, p, t)=e \\ \varepsilon(i, p, t-1)=e}} V(i, p, t)-V(i, p, t-1)
$$

Fluctuations in the traffic demands may occur for a variety of reasons, such as changes in user or application behavior, adaptations caused by end-to-end congestion control, or even routing changes in other domains.

The routing variation $\Delta R(i, e, t)$ considers the destination prefixes that shifted to egress point $e$ during time interval $t$ or shifted from $e$ to another egress point in $t$ :

$$
\Delta R(i, e, t)=\sum_{\substack{p \in P \\ \varepsilon(i, p, t)=e \\ \varepsilon(i, p, t-1) \neq e}} V(i, p, t)-\sum_{\substack{p \in P \\ \varepsilon(i, p, t) \neq=\\ \varepsilon(i, p, t-1)=e}} V(i, p, t-1)
$$

Note that if a routing change occurs within the time interval $t$, we associate all of the traffic associated with that prefix in that time interval with the new egress point.

Not all traffic matrix elements carry the same volume of traffic, and the volume of traffic from an ingress to an egress PoP varies over time. How do we judge if a change in 
the traffic is "large"? There is no absolute standard: one approach might be to judge the size of the change in traffic matrix element relative to the average traffic for that element. However, this is not useful here, because the traffic process itself is non-stationary. It has daily and weekly cycles, as well as level shifts resulting from routing changes. The relative change $\Delta \mathcal{T} M(i, e, t) / \mathcal{T} M(i, e, t)$ (or $\Delta \mathcal{T} M(i, e, t) / \max (\mathcal{T} M(i, e, t), \mathcal{T} M(i, e, t-$ $1))$ ) seems appealing. However, this metric places too much emphasis on large relative changes to small values; for example, a traffic matrix element with $1 \mathrm{kbit} / \mathrm{sec}$ might easily experience a $50 \%$ relative change in traffic without having any significant effect on the network. An alternative metric would be the absolute change $\Delta \mathcal{T} M(i, e, t)$. However, a shift of (say) $10 \mathrm{MB} / \mathrm{sec}$ may be significant for one ingress point but not for another. Another option would be to normalize the value of $\Delta \mathcal{T} M(i, e, t)$ by the total traffic entering ingress point $i$ at time $t$, which would capture changes in the fraction of the incoming traffic that uses a particular egress point. However, this metric depends on the "current" traffic demand at ingress $i$ (which could be low at certain times) and may not accurately reflect the strain imposed on the network by the traffic change. Another extreme approach would be to consider the capacity of the network, and define as large any traffic shift that causes a link to be overloaded. Besides being difficult to compute, this metric is too closely tied to the current design of the network, and is not useful for most typical applications of the traffic matrix such as capacity planning or anomaly detection. Instead, we want a metric that captures properties of the traffic matrix itself, such as how large the traffic changes are relative to the normal variations of traffic matrix elements.

For that, we should consider what type of process we observe, namely, a difference process. Over short time periods, we can approximate the traffic with a linear process $y_{t}=\alpha+\beta t+x_{t}$, where $x_{t}$ is a zero-mean stochastic process, with variance $\sigma^{2}$. We observe the differences $\Delta y_{t}=y_{t}-y_{t-1}$, which will form a stationary process, with mean $\beta$ and variance $2 \sigma^{2}$. Thus we can approximate the difference process by a stationary process, and measure deviations from the mean, relative to the standard deviation of this process. We measure $2 \sigma(i, e)^{2}$ on the traffic variation process $\Delta L(i, e, \cdot)$ (using the standard statistical estimator), and use this to normalize the traffic variations, i.e. we then observe $\Delta \tilde{L}(i, e, t)=\Delta L(i, e, t) / \sqrt{2} \sigma(i, e)$, and $\Delta \tilde{R}(i, e, t)=\Delta R(i, e, t) / \sqrt{2} \sigma(i, e)$.

If the variance of the process $x_{t}$ was time dependent, it might make sense to use a moving average to estimate the process variance at each point in time, i.e. $\sigma(i, e, t)^{2}$, and use this to normalize the traffic variations. We tried such an approach, but it made little difference to the results, and so we use the simpler approach described above.

Figure 3 presents a scatter plot of $\Delta \mathcal{T} \tilde{M}(i, e, t)$ versus $\Delta \tilde{R}(i, e, t)$ for all the valid measurement intervals $t$. The high density of points close to zero shows that large traffic variations are not very frequent $(99.88 \%$ of the traffic variations are in the $[-4,4]$ range). Points along the horizontal line with $\Delta \tilde{R}(i, e, t)=0$ correspond to traffic variations that are not caused by routing changes, whereas points along the diagonal line correspond to variations caused almost exclusively by routing changes. Points in the middle are caused by a mixture of routing changes and load variation. Figure 3 shows that both load and routing are responsible for some big variations. Routing changes, 
however, are responsible for the largest traffic shifts. Indeed, one egress-point change made a traffic matrix element vary more than 70 times the standard deviation.

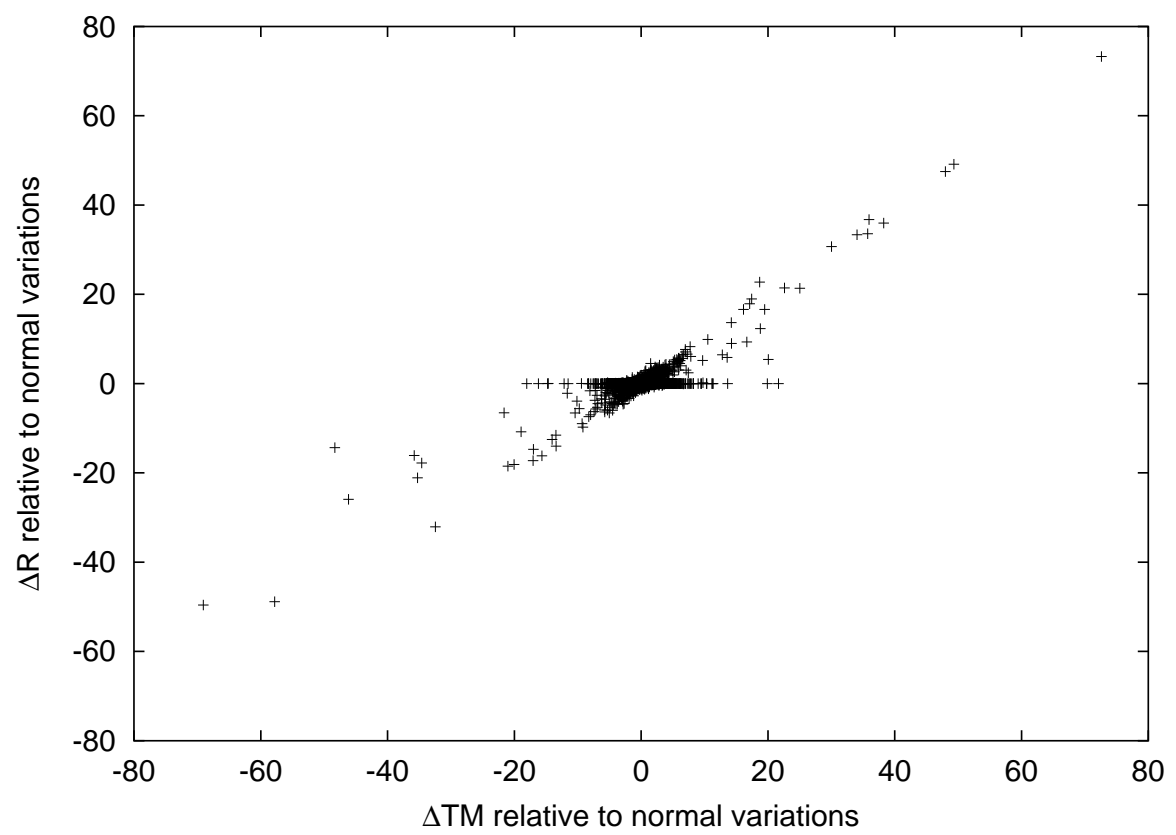

Fig. 3. Scatter plot of $\Delta \tilde{T} \tilde{M}$ versus $\Delta \tilde{R}$ for all traffic matrix elements over the seven-month period.

\subsection{Internal vs. External Routing Changes}

The prefix-to-egress mapping $\varepsilon$ may change because of either internal or external routing events. External routing changes represent any changes in the set of egress points that an AS uses to reach a destination prefix. For example, in Figure 1, the neighbor AS might withdraw the route for $p 2$ from the router $e$, resulting in a change in $\varepsilon$. External routing changes may be caused by a variety of events, such as an internal routing change in another domain, a modification to the local BGP routing policy, or a failure at the edge of the network. In contrast, internal routing changes stem from changes in the routing inside the AS, due to equipment failures, planned maintenance, or traffic engineering. These events affect the prefix-to-egress mapping because the intradomain path costs play a role in the BGP decision process through the common practice of hot-potato routing.

When selecting a best BGP route, a router first considers BGP attributes such as local preference, AS path length, origin type, and the multiple exit discriminator. If 
multiple "equally good" routes remain, the router selects the route with the "closest" egress point, based on the intradomain path costs. Since large ISPs typically peer with each other in multiple locations, the hot-potato tie-breaking step almost always drives the final routing decision for destinations learned from peers, although this is much less common for destinations advertised by customers. In the example in Figure 1, an internal link failure might make router $i$ 's intradomain path cost to $e$ suddenly larger than the path to $e^{\prime}$. This would change the prefix-to-egress mapping for $p 2$, causing a shift in traffic from egress point $e$ to $e^{\prime}$. Using the methodology described in [10], we identified which changes in $\varepsilon$ were caused by internal events.

Figure 4 shows the cumulative distribution functions of $\Delta \tilde{R}$ caused by hot-potato routing and by external BGP changes. For comparison, we also present the cumulative distribution function (CDF) of a normal distribution, which is drawn from randomly generated Gaussian data with standard deviation equal 1, because $\Delta \tilde{R}$ has been normalized to have standard deviation equal 1 . Although the routing events are rare (only $0.66 \%$ of non-zero $\Delta \mathcal{T} M$ are caused by eBGP changes and $0.1 \%$ by hot-potato changes), this result shows that there are significant cases where these events are big, to very big. In particular, approximately $5 \%$ of traffic shifts caused by hot-potato routing are at least one order of magnitude bigger than normal variations. A single internal change is more likely to affect a large number of destination prefixes [10], including the popular destinations receiving large amounts of traffic.

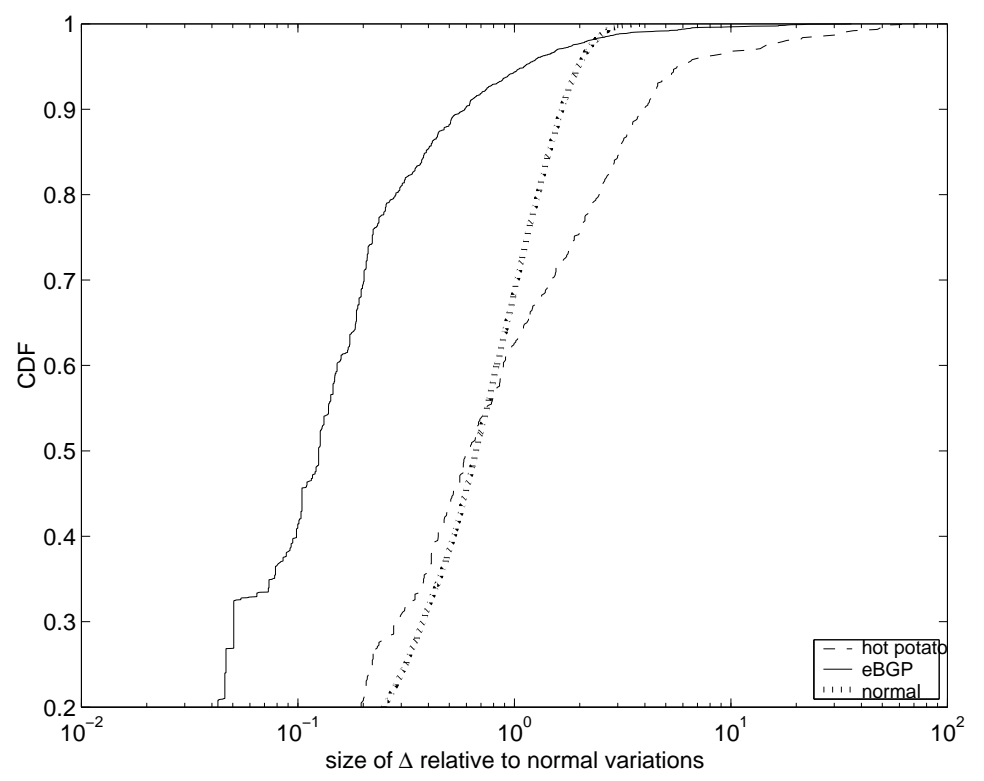

Fig. 4. Cumulative distribution function of $\Delta \tilde{R}$ caused by hot-potato routing and eBGP. 
We analyzed the source of traffic variation for individual traffic matrix elements, and saw that the likelihood of changes in the prefix-to-egress mappings can vary significantly from one ingress router to another. Figures 6 and 5 present the same data as in Figure 3 for two sample traffic matrix elements (Note that the axis are different across the two graphs.). Some traffic matrix elements have no traffic variation caused by routing changes (Figure 5), whereas other have few very large egress shifts (Figure 6). We computed the percent of the traffic matrix elements $(i, e)$ that have large to very large traffic shifts. We define large as more than 4 times the normal traffic variations for $(i, e)$ and very large more than 10 times. Approximately $25 \%$ of ingress-egress pairs $(i, e)$ in our study have no large traffic variation, and the vast majority of them $(85.7 \%)$ have no very large traffic variation. The differences across the traffic matrix elements have two main explanations:

- Size of traffic matrix element. Some traffic matrix elements carry little traffic. Most of the traffic from an ingress router exits the network at few egress PoPs, because of hot-potato routing. For instance, most of the traffic entering in San Diego is likely to stay in the west cost. Therefore, the traffic element San Diego to New York carries very little traffic at any time.

- Impact of internal events. The likelihood of hot-potato routing changes varies significantly from one ingress point to the other [10], depending on the location in the network and the proximity to the various egress points. For our eight ingress points, the fraction of BGP routing changes caused by internal events varies from $1 \%$ to $40 \%$. As a result, the likelihood of large traffic shifts caused by hot-potato routing varies significantly from one traffic matrix element to another.

Out of the traffic matrix elements that do experience large traffic variations $15 \%$ have an average of more than one large traffic variation per week. The small percentage of elements that experience large traffic variations combined with the low frequency large shifts per element may lead to the incorrect conclusion that these events are irrelevant. However, if we consider the network-wide frequency of large traffic shifts, these events happen fairly often. To show this, we have counted the number of 10-minute measurement intervals for which at least one of our eight vantage points experienced a large traffic variation. On average, the network experiences a large traffic variation every four and half hours. Large traffic variations caused by routing changes happen every 2.3 days, and very large routing-induced traffic variations happen every 5.9 days. If our analysis considered all of the PoPs in the network, the overall frequency of large traffic variations would be even higher.

\section{Implication for Traffi c Matrix Studies}

Our analysis on traffic matrix variations has important implications for the results of previous measurement studies.

Differences across vantage points: The results in Section 3 show that the likelihood of changes in the prefix-to-egress mappings can vary significantly from one ingress router to another. In particular, some ingress points may be much more susceptible to hot-potato routing changes than others [10], making analysis of routing stability 


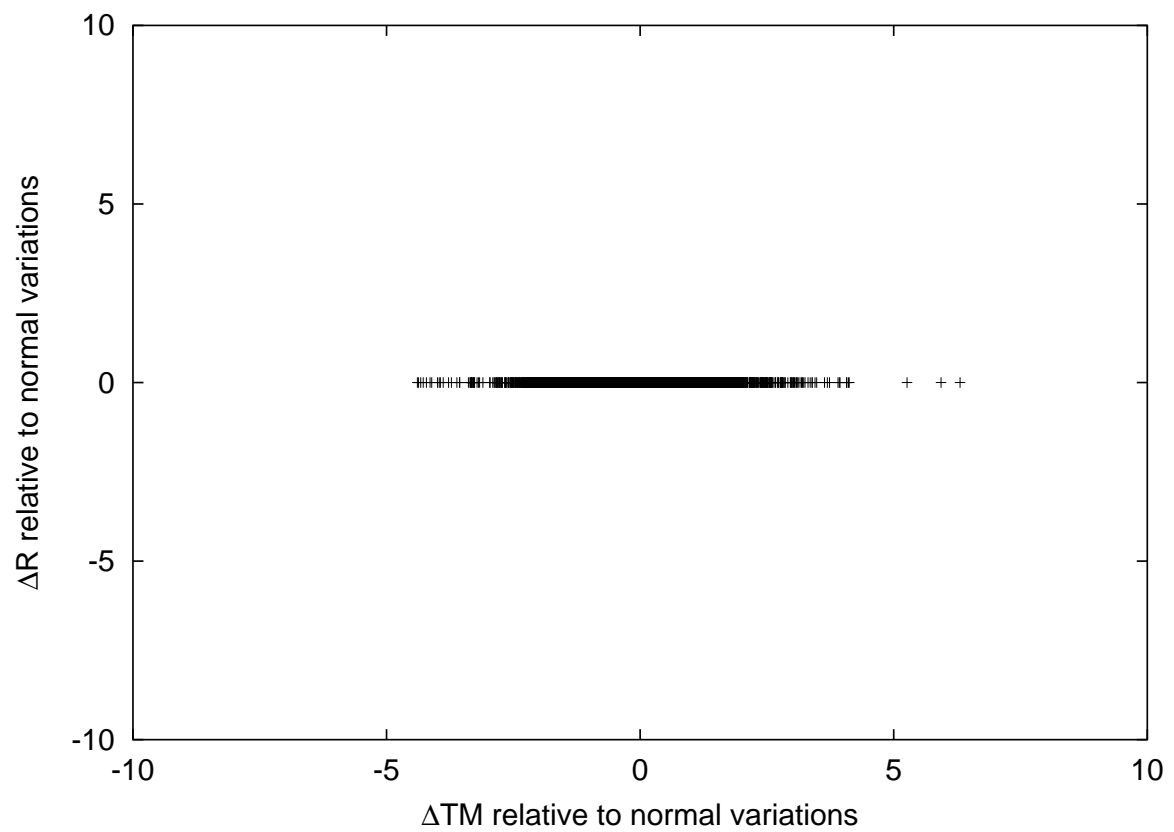

Fig. 5. Scatter plot of $\Delta \tilde{\mathcal{T}} \tilde{M}$ versus $\Delta \tilde{R}$ for a traffic matrix element that have no routing-induced traffic variations over the seven-month period.

very dependent on where the data are collected. For example, the study in [11] showed that popular destination prefixes do not experience BGP routing changes for days or weeks at a time. In addition to studying RouteViews and RIPE BGP feeds, the analysis included iBGP data from two of the eight routers used in our current study. In our analysis, these two routers did not experience many hot-potato routing changes. Had the analysis in [11] analyzed a router that experiences several hot-potato routing changes a day, the conclusions might have been quite different. In fact, hot-potato routing changes can affect a large number of prefixes [10], both popular and not, so we might reasonably expect popular destinations to experience changes in their egress points. A preliminary analysis across all eight vantage points confirms that popular destination prefixes have more BGP instabilities from vantage points that experience more hot-potato routing changes.

Choice of metrics in studying unlikely events: The analysis in Section 3 shows that large changes in the traffic matrix elements occur relatively infrequently. In addition, most changes in the prefix-to-egress mapping do not lead to large traffic shifts, consistent with the results in [7]. Yet, these two results do not imply that routing changes are not a significant contributor to large changes in the traffic matrix elements. In fact, the opposite is true. A small number of routing changes are indeed responsible for a relatively large fraction of the (few) large traffic shifts. In addition, long traces are nec- 


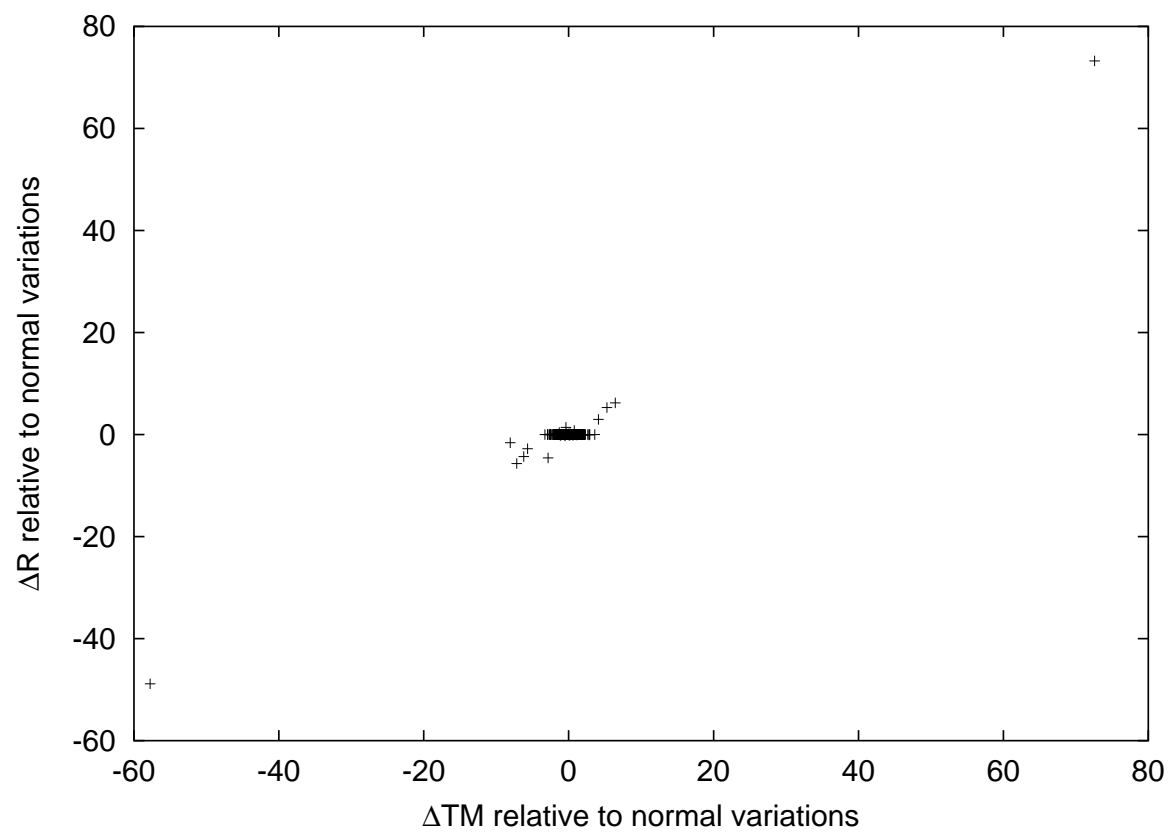

Fig. 6. Scatter plot of $\Delta \tilde{\mathcal{T} M}$ versus $\Delta \tilde{R}$ for a traffic matrix element that has few very large routing-induced traffic shifts over the seven-month period. One traffic shift was over 70 times normal traffic variations!

essary to draw conclusions about infrequent (yet significant) events. The study in [7] draws on five traces of 6-22 hours in duration, outside of the maintenance periods where operators made planned changes to the internal network, making it difficult to conclude definitively if large traffic shifts occur and whether routing contributes to them.

Errors from ignoring egress changes in traffic matrix analysis: Previous work on measuring and analyzing traffic matrices has assumed that routing is stable, in part because fine-grained routing data is sometimes difficult to collect. Most of the work on traffic matrix estimation $[1,2,4]$ assumes that there are no changes in the prefix-toegress mapping or the intradomain paths between the ingress and egress points. Even work on direct measurement of the traffic demands $[5,13]$ has used only daily routing snapshots, although the work in [7] is a notable exception. Using out-of-date routing information runs the risk of associating some traffic measurements with the wrong elements in the traffic matrix. In some cases, the routing changes might lead to secondorder effects on the traffic (e.g., by causing congestion or increasing the round-trip time) that may appear in the data, but the primary affect of the traffic moving to a different egress point is obscured-as is the reason for the variation in the traffic. In addition, changes in the prefix-to-egress mapping may cause large fluctuations in multiple traffic matrix elements at the same time, which would be obscured if the traffic matrix is com- 
puted or analyzed without regard for routing changes. In our ongoing work, we plan to quantify the errors in the traffic matrix computed using daily snapshots, similar to the approach in [7] but focusing specifically on routing changes that have a large affect on multiple traffic matrix elements.

Dependence on network design, traffic, and goals: The results of any traffic matrix analysis, including ours, depend on the details of the network under study. For example, large ISP networks handle high volumes of aggregated traffic, which may experience much smaller statistical fluctuations in the traffic. In addition, a large ISP network connects to its peers and many of its customers in multiple locations in the network, increasing the likelihood that destination prefixes are reachable via multiple egress points. This makes an ISP network much more likely to experience changes in the prefix-to-egress mapping over time. Together, these two factors tend to make routing changes have a larger relative influence on the traffic matrix in ISP networks than in other kinds of networks. Even within a single network, the fluctuations in the traffic matrix may vary from one ingress point to another, due to hot-potato routing changes or the particular senders and receivers connected to that router. Identifying metrics that isolate each of these effects would be very helpful in deepening our fundamental understanding of what causes fluctuations in traffic matrices.

\section{Conclusion}

Our study shows that large traffic variations, while unusual, do sometimes happen. Although most routing changes typically do not affect much traffic, routing is usually a major contributor to large traffic variations. This implies that network operators need to design the network to tolerate traffic variations that are much larger than typical statistical fluctuations in the incoming traffic. In addition, research on traffic engineering and anomaly detection should take into account the impact of routing on the traffic matrix. Since both the traffic demands $V$ and the prefix-to-egress mapping $\varepsilon$ are necessary to compute an accurate traffic matrix, we believe it is more accurate to operate on $V$ and $\varepsilon$ directly, rather than simply on $\mathcal{T} \mathcal{M}$.

This work has implications for both the research and network operations communities. Researchers should consider the impact of changes in the prefix-to-egress mapping when analyzing the traffic matrix. Ignoring these changes might lead to wrong conclusions about traffic matrix stability. Operators need to provision for traffic variations that are much larger than normal traffic fluctuations. In addition, operators often need to diagnose the cause of a large surge in traffic. Our work shows that the routing system is one important place they should look for explanations.

As future work we plan to quantify the inaccuracies introduced in studies of routing and traffic stability when changes in $\varepsilon$ are ignored. We are also studying the duration of the traffic shifts. If traffic shifts are short-lived, then network operators should just over-provision to tolerate them. If they are long-lived, however, adapting the routing protocol configuration may be a better approach for alleviating congestion. 


\section{References}

1. J. Cao, D. Davis, S. V. Wiel, and B. Yu, 'Time-varying network tomography," J. American Statistical Association, December 2000.

2. A. Medina, N. Taft, K. Salamatian, S. Bhattacharyya, and C. Diot, "Traffic matrix estimation: Existing techniques and new directions," in Proc. ACM SIGCOMM, August 2002.

3. Y. Zhang, M. Roughan, N. Duffield, and A. Greenberg, 'Fast, accurate computation of largescale IP traffic matrices from link loads," in Proc. ACM SIGMETRICS, June 2003.

4. Y. Zhang, M. Roughan, C. Lund, and D. Donoho, "An information-theoretic approach to traffic matrix estimation," in Proc. ACM SIGCOMM, August 2003.

5. A. Lakhina, K. Papagiannaki, M. Crovella, C. Diot, E. Kolaczyk, and N. Taft, 'Structural analysis of network traffic flows," in Proc. ACM SIGMETRICS, June 2004.

6. A. Lakhina, M. Crovella, and C. Diot, "Characterization of Network-Wide Anomalies in Traffic Flows," in Proc. Internet Measurement Conference, October 2004.

7. S. Agarwal, C.-N. Chuah, S. Bhattacharyya, and C. Diot, 'Impact of BGP dynamics on intradomain traffic," in Proc. ACM SIGMETRICS, June 2004.

8. Sampled Netflow. http://www.cisco.com/univercd/cc/td/doc/product/ software/ios120/120newfot/120limit/120s/120s11/12s_sanf.htm.

9. A. Shaikh and A. Greenberg, 'OSPF monitoring: Architecture, design, and deployment experience," in Proc. USENIX/ACM NSDI, March 2004.

10. R. Teixeira, A. Shaikh, T. Griffin, and J. Rexford, 'Dynamics of hot-potato routing in IP networks," in Proc. ACM SIGMETRICS, June 2004.

11. J. Rexford, J. Wang, Z. Xiao, and Y. Zhang, 'BGP routing stability of popular destinations," in Proc. Internet Measurement Workshop, November 2002.

12. N. Duffield, C. Lund, and M. Thorup, 'Estimating flow distributions from sampled flow statistics," in Proc. ACM SIGCOMM, August 2003.

13. A. Feldmann, A. Greenberg, C. Lund, N. Reingold, J. Rexford, and F. True, 'Deriving traffic demands for operational IP networks: Methodology and experience," IEEE/ACM Trans. on Networking, June 2001. 